INVESTIGACIÓN

Recibido: 04/02/2019 --- Aceptado: 08/05/2019 --- Publicado: 15/09/2019

\title{
ESTUDIO SOBRE EXPECTATIVAS LABORALES DE ESTUDIANTES DE LA CARRERA DE EDUCACIÓN INICIAL EN LA UNIVERSIDAD METROPOLITANA CARACAS - VENEZUELA
}

\section{Study about labor expectations of students of the initial education career at the Metropolitan University Caracas-Venezuela}

Natalia Castañón Octavio ${ }^{1}$ Universidad Metropolitana. Venezuela. ncastanon@unimet.edu.ve

Laura Ocanto Gómez. Universidad Metropolitana. Venezuela. laura.ocanto@correo.unimet.edu.ve

Valerie Tirado Aldana. Universidad Metropolitana. Venezuela. valerietirado@correo.unimet.edu.ve

\section{RESUMEN}

El presente estudio tuvo como objetivo principal determinar las expectativas laborales de los estudiantes de la carrera de Educación Inicial en la Universidad Metropolitana (Caracas-Venezuela) para contribuir al proceso de mejoramiento continuo de la calidad académica. Para ello se diseñó una investigación no experimental de tipo descriptivo conformada por dos variables: características de los estudiantes de educación superior y expectativas laborales. Tras la aplicación de una encuesta mixta (preguntas abiertas y cerradas) se analizaron los datos de manera cuantitativa y cualitativa. Los resultados más resaltantes señalaron el deseo de los sujetos de convertirse en agentes de cambio y desempeñar su rol docente $(90,24 \%)$ a pesar de considerar que la carrera no es valorada por la sociedad venezolana, la mayoría de la muestra indicó que han desarrollado habilidades de sensibilidad social, fomentada directamente desde la Universidad Metropolitana y se mostró que el 47,62\% como expectativa laboral principal a la Gerencia Educativa, lo cual pareciera indicar las altas expectativas laborales pues existe la proyección en el escalafón más alto de la jerarquía educativa. Se concluyó que estudiar las expectativas laborales de los estudiantes de educación superior contribuye

\footnotetext{
${ }^{1}$ Natalia Castañón Octavio: Profesora e investigadora en la Universidad Metropolitana. Licenciada en Educación (Universidad Metropolitana, 1991). Maestría en Psicología Escolar (Universidad Complutense de Madrid, 1993). Doctorada en Filosofía y Ciencias de la Educación (Universidad Complutense de Madrid, 1999).
} 
Castañón Octavio, N.; Ocanto Gómez, L., y Tirado Aldana, V. Estudio sobre expectativas laborales de estudiantes de la carrera de educación inicial en la Universidad Metropolitana de

Caracas-Venezuela

al mejoramiento de los pensum de estudio lo cual se traduce en un mejor servicio y aumento de la calidad académica, en el marco que conocer la realidad del sector sociallaboral en conjunto con los intereses, necesidades y expectativas de los sujetos, favorece la inserción en el mercado laboral.

PALABRAS CLAVE: expectativas laborales - educación inicial - estudiantes universitarios - trabajo - docencia - sensibilidad social - mercado laboral.

\section{ABSTRACT}

The main objective of this study was to determine the job expectations of the students of the Initial Education career at the Metropolitan University (Caracas-Venezuela) to contribute to the process of continuous improvement of academic quality. To this end, a non-experimental descriptive research was designed, consisting of two variables: characteristics of higher education students and job expectations. After the application of a mixed survey (open and closed questions) the data were analyzed in a quantitative and qualitative way. The most outstanding results indicated the desire of the subjects to become agents of change and to fulfill their teaching role $(90.24 \%)$ Despite considering that the race is not valued by Venezuelan society, most of the sample indicated that they have developed skills of social sensitivity, fostered directly from the Metropolitan University and showed that $47.62 \%$ as the main job expectation to the Educational Management, which seems to indicate the high expectations of work as there is a projection in the highest hierarchy of the educational hierarchy. It was concluded that studying the work expectations of higher education students contributes to the improvement of study plans, which translates into the improvement of the academic quality service, within the framework of knowing the reality of the social-labor sector in conjunction with the interests, needs and expectations of subjects of higher education that favor the insertion in the labor market.

KEY WORDS: labor expectations - initial education - university students - work teaching - social sensitivity - labor market.

\section{ESTUDOS SOBRE AS EXPECTATIVAS LABORAIS DOS ESTUDANTES QUE CURSAM EDUCAÇÃO INICIAL NA UNIVERSIDADE METROPOLITANA DE CARACAS - VENEZUELA}

\section{RESUME}

Este estudo teve como principal objetivo determinar as expectativas laborais dos estudantes que cursam educação inicial na Universidade Metropolitana de Caracas (Venezuela) para contribuir ao processo de melhoramento continuo da qualidade acadêmica para isso se desenhou uma investigação não experimental tipo descritiva conformada por duas

Vivat Academia. Revista de Comunicación. 15 septiembre 2019 /15 diciembre 2019, n 148, 57-76 
Castañón Octavio, N.; Ocanto Gómez, L., y Tirado Aldana, V. Estudio sobre expectativas laborales de estudiantes de la carrera de educación inicial en la Universidad Metropolitana de

Caracas-Venezuela

variáveis: características dos estudantes de educação superior e expectativas laborais. Depois de uma encosta mista (perguntas abertas e fechadas) analisaram os dados de maneira quantitativa e qualitativa. Os resultados mais ressaltantes assinalam o desejo dos sujeitos de converter-se em agentes de troca e desempenhar seu papel docente (90,24 por cento) apesar de considerar que o curso não e valorizado pela sociedade venezuelana, a maioria da amostra indicou que desenvolveram habilidades de sensibilidade social, fomentada diretamente desde a Universidade Metropolitana e foi mostrado que um 47,62 por cento como expectativa laboral principal à Gerencia Educativa, o qual parecia indicar as altas expectativas laborais, pois existe a projeção em um escalão mais alto da hierarquia educativa. Concluiu que estudar as expectativas laborais dos estudantes de educação superior contribui ao melhoramento dos planos de estudo o qual se traduz em serviço de qualidade acadêmica, no contexto de conhecer a realidade do setor-sócio laboral em conjunto com os interesses, necessidades e expectativas dos sujeitos, favorecendo a inserção no mercado laboral.

PALAVRAS CHAVE: expectativas laborais - educação inicial - estudantes universitários - trabalho - docência - sensibilidade social - mercado laboral.

\section{Como citar el artículo:}

Castañón Octavio, N.; Ocanto Gómez, L., y Tirado Aldana, V. (2019). Estudio sobre expectativas laborales de estudiantes de la carrera de educación inicial en la Universidad Metropolitana de Caracas-Venezuela. [Study about labor expectations of students of the initial education career at the Metropolitan University CaracasVenezuela]. Vivat Academia. Revista de Comunicación, 148, 57-76.

DOI: $\underline{\text { http://doi.org/10.15178/va.2019.148.57-76 }}$

Recuperado de http://www.vivatacademia.net/index.php/vivat/article/view/1157

\section{INTRODUCCIÓN}

Los estudiantes de la carrera de Educación Inicial de la Universidad Metropolitana (UNIMET), al igual que el común de los estudiantes universitarios, poseen expectativas laborales. Estas expectativas presentan diferentes visiones dependiendo particularmente de cada estudiante.

Los autores están de acuerdo con la definición de expectativas laborales propuesta por Batlle, Vidondo, Dueñas, Nuñez y Rodríguez (2009), al definirlas como "las elecciones que involucran aspectos más realistas, pudiendo reconocer e incluir un cierto conocimiento de sí mismo sobre capacidades e intereses y también aspectos del contexto" (p. 5).

Sin embargo, las expectativas laborales, como se mencionó anteriormente, son particulares en cada estudiante, varían con el tiempo y dependen tanto del entorno

Vivat Academia. Revista de Comunicación. 15 septiembre 2019 /15 diciembre 2019, n 148, 57-76 
Castañón Octavio, N.; Ocanto Gómez, L., y Tirado Aldana, V. Estudio sobre expectativas laborales de estudiantes de la carrera de educación inicial en la Universidad Metropolitana de

Caracas-Venezuela

personal, social, cultural como del nivel económico, etc. Bourdieu citado por Corica (2012), menciona que:

Las aspiraciones tienden a tornarse más realistas, más estrictamente ajustadas a las posibilidades reales, a medida que estas últimas aumentan. La distancia entre el nivel de aspiración y el nivel de realidad, entre las necesidades y los medios, tiende a decrecer a medida que se eleva en el estrato social y, por lo tanto, en el nivel de ingreso (p. 86).

Según Bourdieu, la diferenciación económica va a estar vinculada con las aspiraciones; es decir, que las decisiones futuras se tomarán a partir de las posibilidades efectivas de ser realizadas.

Otro punto de vista, es el presentado por Bandura (2001), quien argumenta que las expectativas laborales, o como él las define expectativas de resultados, dependen de los juicios de valor que cada estudiante tenga sobre sus competencias para desempeñarse en una situación particular. Para el citado autor, las expectativas de resultados se presentan de tres formas diferentes: Las primeras son expectativas materiales, las cuales están vinculadas tanto a los costos, materiales y sus beneficios; como a la retribución económica y estabilidad en el empleo, las segundas son las expectativas sociales que se forman a partir de las reacciones sociales positivas o negativas, como el prestigio, el poder, la influencia, y la aprobación social; y por último, se presentan las expectativas personales encaminadas a las reacciones autoevaluativas negativas y positivas, entre estas se exteriorizan la satisfacción personal, la valoración del empleo y la autorrealización, etc.

Evidentemente, la frustración y ansia causada por el incumpliendo de las expectativas laborales en el país, sumado a otros factores actuales, conlleva a un éxodo profesional que desabastece los principales espacios de formación inicial, en cuanto a personal capacitado para ejercer funciones; bajo su correcta titulación. Lo anterior se fundamenta en las estadísticas presentadas por el Banco Mundial (2017a), las cuales estipulan que el volumen de emigrantes venezolanos para el año 2015 fue de 1.404 .448 millones, lo que equivale al 4,6\% de la población total del país.

Sumado a lo anterior, los incentivos generados por las nuevas oportunidades de trabajo y los altos niveles remunerativos que ofrecen los países desarrollados invitan al éxodo profesional. Dicha afirmación, no sólo se restringe a las características laborales, es necesario incluir las particularidades del entorno socio-cultural y político de una sociedad que trastoca la vida cotidiana de sus integrantes, y que influye notablemente en la toma de decisiones para aspiraciones laborales, familiares y personales (Sieglin y Zúñiga, 2010, p. 58).

Se debe mencionar que, existen otros elementos que incentivan la migración académica, estos pueden ser, el deseo de estudiar en una institución con reconocimiento

Vivat Academia. Revista de Comunicación. 15 septiembre 2019 / 15 diciembre 2019, nº 148, 57-76 
Castañón Octavio, N.; Ocanto Gómez, L., y Tirado Aldana, V. Estudio sobre expectativas laborales de estudiantes de la carrera de educación inicial en la Universidad Metropolitana de

Caracas-Venezuela

y prestigio internacional. Según Gérard, Kail, Lanoue, Proteau y Ronan (2008) citado por Sieglin y Zúñiga (2010), este tipo de instituciones de educación superior, que de permitirle formar parte de una comunidad científica internacional y proporcionar el acceso a otras culturas, lo puede catapultar en un profesional exitoso y reconocido internacionalmente con el único fin de garantizar el nivel salarial deseado (p. 61).

Habría que señalar también, que la inserción laboral es la meta primordial de los estudiantes universitarios. Al respecto Pérez (2015), señala que:

La inserción laboral de los recién graduados es un proceso complejo, muy dependiente de la evolución del mercado laboral, el cual está caracterizado, en general, por una menor estabilidad de empleo, mayor precariedad laboral, un aumento de la competitividad, mayores exigencias de cualificación para el desempeño de puestos, entre otros (p. 7).

En cuanto a la carrera de Educación, puede estar presentándose una desinformación y degradación de la importancia de la profesión docente. Lo anterior, influye directa y negativamente en el interés de los nuevos estudiantes a la hora de considerarla como su futura profesión, al creer que no cumplirá con sus expectativas laborales. Al respecto, Caram, Gil y Naigeboren (2009) señalan que:

Existe una ambigüedad con respecto a las expectativas profesionales al ingresar en la carrera, se observa que en los primeros años sólo la minoría vincula el rol del pedagogo con la función docente, mientras que en el último tramo de la carrera las expectativas profesionales se amplían y se definen hacia otros campos de inserción laboral. [...] La ambigüedad podría estar relacionada con falta de información o desconocimiento acerca de los roles profesionales, con aspectos motivacionales y de elección vocacional. Sin duda la crisis económica global, la desocupación, la inestabilidad del mundo laboral, la dificultad para encontrar un trabajo y mantenerlo, son factores que inciden en esta endeble identidad ocupacional. (p. 338).

Diferentes países han implementado como respuesta a lo anterior, los llamados Observatorios, los cuales, como estipula Farné (2011) sirven como un "instrumento de permanente medición e investigación de los principales indicadores sociales, económicos y en especial de la situación del mercado de trabajo y formativo a nivel regional" (p. 4). De esta manera se fortalece, como plantea Pérez (2015), un "binomio universidad - mercado, en términos de ajuste entre oferta de titulados y demandas del mercado laboral" (p. 11).

En referencia a la carrera de Educación Inicial, esta se fundamenta en el ejercicio de la docencia, la investigación, la promoción cultural, el desarrollo social-comunitario y la

Vivat Academia. Revista de Comunicación. 15 septiembre 2019 /15 diciembre 2019, n 148, 57-76 
Castañón Octavio, N.; Ocanto Gómez, L., y Tirado Aldana, V. Estudio sobre expectativas laborales de estudiantes de la carrera de educación inicial en la Universidad Metropolitana de

Caracas-Venezuela

administración de la educación, siendo estas las intenciones principales de su formación. La situación venezolana, en cuanto a temas de educación, invita a gestionar un cambio en la visión pedagógica, donde la práctica educativa posea un sentido crítico, innovador y autogestionario del hecho educativo y social (Unimet, s.f.). Para la Universidad Metropolitana en armonía con su misión y visión, las competencias específicas en la formación de sus docentes son:

- Planificación de la educación: formación profesional desde el ámbito curricular e institucional.

- Evaluación: formación profesional para la supervisión y el seguimiento de procesos sociales y educativos, a fin de lograr su pertinencia.

- Investigación en dos dimensiones: integrada al acto docente y como actividad científica.

- Competencia del docente como agente del cambio social en tres dimensiones: educativa, legal y cultural.

La carrera de Educación tiene tres diferencias con relación a otras carreras en la misma institución. En primer lugar, se tiene la posibilidad de obtener el título de Técnico Superior en Educación, donde se cursan siete trimestres de la carrera con requisitos obligatorios como: prelatoria y servicio comunitario, prácticas docentes, nivel de inglés certificado por la universidad como bilingüe y seis asignaturas electivas. En segundo lugar, la modalidad de estudio es de Licenciado en Educación con tres menciones: Inicial, Integral y Sin Mención. En tercer lugar, su flujograma está conformado por módulos fomentando la educación integral y la autogestión (Unimet, s.f).

No obstante, lo anterior, en la literatura consultada no se encontró evidencia de investigaciones previas, en las cuales se determinen las expectativas laborales de los estudiantes de Educación Inicial en UNIMET.

Respondiendo a los resultados de la investigación, se desea argumentar la siguiente interrogante: ¿Cuáles son las expectativas laborales de los estudiantes de Educación Inicial en Universidad Metropolitana?

\section{OBJETIVOS}

\subsection{Objetivo general}

Determinar las expectativas laborales de los estudiantes de la carrera de Educación Inicial en la UNIMET para contribuir al proceso de mejoramiento continuo de la calidad académica.

\subsection{Objetivos específicos}

1. Detectar las expectativas laborales de los estudiantes de la carrera Educación Inicial de la Universidad Metropolitana, según las características personales.

Vivat Academia. Revista de Comunicación. 15 septiembre 2019 /15 diciembre 2019, n 148, 57-76 
Castañón Octavio, N.; Ocanto Gómez, L., y Tirado Aldana, V. Estudio sobre expectativas laborales de estudiantes de la carrera de educación inicial en la Universidad Metropolitana de

Caracas-Venezuela

2. Caracterizar las expectativas laborales de los estudiantes de la carrera Educación Inicial de la Universidad Metropolitana, según el perfil académico.

3. Explorar las expectativas laborales de los estudiantes de la carrera Educación Inicial de la Universidad Metropolitana, según el contexto país.

\section{METODOLOGÍA}

\subsection{Diseño de la investigación}

La presente investigación se basa en un diseño no experimental, ya que se realiza sin manipular deliberadamente variables. Se estudia el fenómeno de las expectativas laborales de los estudiantes de Educación Inicial de la UNIMET, y posteriormente se analizan los datos obtenidos. Identificando los elementos del estudio no experimental, debido a que no se genera ningún evento, sino que se observan situaciones ya existentes, sin ser provocadas intencionalmente; por quien realiza la investigación, no se tiene control directo ni se puede influir sobre ellas, porque ya sucedieron, al igual que sus efectos (Hernández, Fernández y Baptista, 2010).

Se puede definir como un estudio transversal, debido a que los datos se recolectan en un único momento (Hernández, Fernández y Baptista, 2010), siendo este el trimestre correspondiente a abril-julio del 2018.

\subsection{Tipo de investigación}

Corresponde a un tipo de investigación descriptivo, que "busca especificar las propiedades, las características y los perfiles de personas, grupos, comunidades, procesos, objetos o cualquier otro fenómeno que se someta a un análisis" (Hernández, Fernández y Baptista, 2010, p. 80). Con este estudio se busca determinar características, perfiles y tendencias laborales del grupo de los estudiantes de Educación Inicial de la UNIMET, no se pretende indicar efectos pero si indagar posibles relaciones.

\subsection{Variables, dimensiones e indicadores}

Las variables de la presente investigación, se centran en los estudiantes de educación superior, específicamente aquellos que pertenecen a la carrera de Educación Inicial de la UNIMET, y sus expectativas laborales, ambas sin recibir manipulación por parte de las investigadoras, buscando clarificar la relación entre ambas.

Es de suma importancia, establecer el concepto de variable, el cual, es brindando por Balestrini (2002) como "un aspecto o dimensión de un objeto, o una propiedad de estos aspectos o dimensiones que adquiere distintos valores y por lo tanto varía" (p. 113).

La variable dependiente estudiantes universitarios es, según la Facultad de Ciencias Jurídicas y Políticas de la Universidad del Zulia (2013):

Vivat Academia. Revista de Comunicación. 15 septiembre 2019 /15 diciembre 2019, n 148, 57-76 
Castañón Octavio, N.; Ocanto Gómez, L., y Tirado Aldana, V. Estudio sobre expectativas laborales de estudiantes de la carrera de educación inicial en la Universidad Metropolitana de

Caracas-Venezuela

Estudiantes de educación universitaria quienes hayan obtenido el título de bachiller y cumplan los requisitos de ingreso a las instituciones de educación universitaria establecidos por ellas y por los órganos del subsistema de educación universitaria dentro de sus áreas de competencia. (s.p.)

La variable dependiente expectativas laborales, se definen como un conjunto de expectativas personales, materiales y sociales, que van ajustadas a la realidad socioeconómica de sus autores, donde existe una satisfacción material y social, mediante el cumplimiento de las mismas. (Suriá et al., 2013b).

Las variables, dimensiones e indicadores de esta investigación se presentan en el cuadro a continuación:

Tabla 1. Variables, dimensiones e indicadores.

\begin{tabular}{|c|c|c|}
\hline Variables & Dimensiones & Indicadores \\
\hline \multirow{2}{*}{$\begin{array}{l}\text { Estudiantes } \\
\text { universitarios } \\
\text { de la carrera } \\
\text { Educación } \\
\text { Inicial de la } \\
\text { UNIMET }\end{array}$} & Perfil Personal & $\begin{array}{l}\text { Género } \\
\text { Nivel de ingresos: método de pago de la matrícula, ocupación } \\
\text { del responsable económico, vivienda, estratos. } \\
\text { Intereses personales. }\end{array}$ \\
\hline & Perfil Académico & $\begin{array}{l}\text { Índice académico. } \\
\text { Competencias específicas. } \\
\text { Oportunidades de empleo. } \\
\text { Vías de ingreso }\end{array}$ \\
\hline \multirow{5}{*}{$\begin{array}{l}\text { Expectativas } \\
\text { Laborales }\end{array}$} & Materiales & $\begin{array}{l}\text { Remuneración salarial. } \\
\text { Cargo a desempeñar. } \\
\text { Lugar de trabajo: centros educativos privados o públicos, } \\
\text { organizaciones, etc. }\end{array}$ \\
\hline & Sociales & $\begin{array}{l}\text { Reputación. } \\
\text { Cargo a desempeñar. } \\
\text { Lugar de trabajo. }\end{array}$ \\
\hline & $\begin{array}{l}\text { Realización } \\
\text { Personal }\end{array}$ & $\begin{array}{l}\text { Altruismo. } \\
\text { Cargo a desempeñar. } \\
\text { Lugar de Trabajo }\end{array}$ \\
\hline & Contexto país & $\begin{array}{l}\text { Venezuela } \\
\text { Exterior del país. }\end{array}$ \\
\hline & \begin{tabular}{|l|} 
Condiciones \\
Laborales \\
\end{tabular} & $\begin{array}{l}\text { Contrato: remuneración salarial, beneficios. } \\
\text { Auto actualización }\end{array}$ \\
\hline
\end{tabular}

Fuente: Elaboración propia.

\subsection{Población y muestra}

La población de este estudio, está constituida por los estudiantes de la carrera de Educación Inicial de la Universidad Metropolitana, siendo un total de treinta y tres (33)

Vivat Academia. Revista de Comunicación. 15 septiembre 2019 /15 diciembre 2019, n 148, 57-76 
Castañón Octavio, N.; Ocanto Gómez, L., y Tirado Aldana, V. Estudio sobre expectativas laborales de estudiantes de la carrera de educación inicial en la Universidad Metropolitana de

Caracas-Venezuela

estudiantes. Se utilizó el muestreo intencional por conveniencia. El criterio establecido para la muestra era ser estudiantes activos que cursen asignaturas correspondientes al plan de estudios de Educación Inicial, en la modalidad de pregrado.

\subsection{Instrumento de recolección y análisis de los datos}

El instrumento de recolección, constituye la principal fuente de obtención de los datos, los cuales se fundamentan en las variables estudiadas, este arroja resultados de carácter cuantitativo, pero a su vez, la medición de resultados cualitativos.

La recolección de los datos basados en las variables, siendo éstas, los estudiantes universitarios y sus expectativas laborales, se realizó a través de un cuestionario, cuya modalidad de encuesta se realizó de forma escrita mediante un instrumento o formato en papel contentivo de una serie de preguntas. Se le denomina cuestionario auto administrado porque debe ser llenado por el encuestado, sin intervención del encuestador.

La encuesta utilizada fue adaptada a partir del instrumento elaborado para la investigación en curso realizada por el equipo de investigación de la profesora Natalia Castañón Octavio denominada Expectativas Laborales en Educación Superior.

Previo a la aplicación del cuestionario, se llevó a cabo la validación del instrumento por cuatro profesores de la Universidad Metropolitana y se realizó una prueba piloto con estudiantes.

Este estudio se caracteriza por un enfoque de análisis cuantitativo, ya que los datos recolectados mediante la encuesta, son resultados del instrumento de medición. Además, de sus características cualitativas por la descripción de las expectativas laborales identificadas. Se utilizará el método mixto ya que brinda una perspectiva más amplia del fenómeno a estudiar.

\section{RESULTADOS}

A continuación se dará a conocer los resultados y posteriormente se describirán algunas posibles relaciones que se desprenden de los mismos. La encuesta fue entregada a 33 sujetos que conformaban la muestra, sin embargo respondieron 21 sujetos.

Tabla 2. Índice Académico Acumulado (IAA).

\begin{tabular}{|l|c|c|}
\hline Opciones & Frecuencia & Porcentaje \\
\hline De 10 a 11,9 puntos & 0 & $0 \%$ \\
\hline De 12 a 13,9 puntos & 4 & $19,05 \%$ \\
\hline De 14 a 15,9 puntos & 11 & $52,38 \%$ \\
\hline
\end{tabular}

Vivat Academia. Revista de Comunicación. 15 septiembre 2019 /15 diciembre 2019, n 148, 57-76 
Castañón Octavio, N.; Ocanto Gómez, L., y Tirado Aldana, V. Estudio sobre expectativas laborales de estudiantes de la carrera de educación inicial en la Universidad Metropolitana de

Caracas-Venezuela

\begin{tabular}{|l|c|c|}
\hline De 16 a 17,5 puntos & 3 & $14,29 \%$ \\
\hline De 17,5 a 18,5 puntos & 2 & $9,52 \%$ \\
\hline De 18,5 a 20 puntos & 1 & $4,76 \%$ \\
\hline Respuestas obtenidas & 21 & \\
\hline
\end{tabular}

Fuente: Elaboración propia.

El Índice Académico Acumulado (IAA) es una apreciación general del rendimiento, donde la sumatoria de las ponderaciones obtenidas ubica al estudiante en un determinado puntaje. Tal como se muestra en la tabla, el 52,38\% de los estudiantes posee un IAA entre 14 y 15,9 puntos. Un 19,05\% entre 12 y 13,9 puntos. 12 Sujetos no respondieron a esta pregunta.

Tabla 3. En caso de haber entrado a través de la Prueba Diagnóstica Universitaria (PDU), especifique en qué lista se posicionó.

\begin{tabular}{|l|c|c|}
\hline Opciones & Frecuencia & Porcentaje \\
\hline Lista 1 & 4 & $19,05 \%$ \\
\hline Lista 2 & 5 & $23,81 \%$ \\
\hline Lista 3 & 2 & $9,52 \%$ \\
\hline Lista 4 & 5 & $23,81 \%$ \\
\hline Lista 5 & 1 & $4,76 \%$ \\
\hline Respuestas obtenidas & 17 & \\
\hline
\end{tabular}

Fuente: Elaboración propia.

En vista que "lista 1" se refiere a alto rendimiento académico en las áreas instrumentales de "matemática" y "lenguaje", se puede evidenciar que la mayoría del grupo entró a la Universidad con debilidades de estas áreas. Estos resultados se relacionarán con los Índices Académicos Actuales (IAA), para verificar el progreso de cada uno de los sujetos.

Tabla 4. Puntualice su oportunidad de trabajo deseada.

\begin{tabular}{|l|c|c|}
\hline Opciones & Frecuencia & Porcentaje \\
\hline Docente & 7 & $33,33 \%$ \\
\hline Psicopedagogo & 1 & $4,76 \%$ \\
\hline Gerente educativo & 10 & $47,62 \%$ \\
\hline Dueño de preescolar & 2 & $9,52 \%$ \\
\hline Decano & 1 & $4,76 \%$ \\
\hline Respuestas obtenidas & 21 & \\
\hline
\end{tabular}

Fuente: Elaboración propia.

Vivat Academia. Revista de Comunicación. 15 septiembre 2019 /15 diciembre 2019, nº 148, 57-76 
Castañón Octavio, N.; Ocanto Gómez, L., y Tirado Aldana, V. Estudio sobre expectativas laborales de estudiantes de la carrera de educación inicial en la Universidad Metropolitana de Caracas-Venezuela

Los resultados evidencias dos focos principales como trabajos deseados. En primer lugar la gerencia educativa y a continuación.

Tabla 5. Áreas en la que desea desempeñar al obtener el título de Licenciado en Educación Inicial.

\begin{tabular}{|l|c|c|}
\hline Opciones & Frecuencia & Porcentaje \\
\hline $\begin{array}{l}\text { Docencia en los diferentes niveles del } \\
\text { sistema escolar }\end{array}$ & 20 & $95,24 \%$ \\
\hline $\begin{array}{l}\text { Capacitación, investigación, coordinación y } \\
\text { gerencia de centros educativos. }\end{array}$ & 11 & $52,38 \%$ \\
\hline Consultor multiorganizacional & 3 & $14,29 \%$ \\
\hline $\begin{array}{l}\text { Diseño de programas educativos en } \\
\text { multimedia }\end{array}$ & 3 & $14,29 \%$ \\
\hline Promoción comunitaria & 2 & $9,52 \%$ \\
\hline Evaluación y diseño curricular & 1 & 4,76 \\
\hline Respuestas obtenidas & 40 & \\
\hline
\end{tabular}

Fuente: Elaboración propia.

Ante esta pregunta los sujetos podían responder a varias alternativas, donde se evidencia en sus resultados que casi toda la muestra desea desempeñarse como docente y la mitad de la misma como gerente educativo. Ello coincide con los resultados señalados en la tabla 3.

Tabla 6. La carrera que está estudiando le ayudará a conseguir su trabajo anhelado.

\begin{tabular}{|l|c|c|}
\hline Opciones & Frecuencia & Porcentaje \\
\hline Sí & 21 & $100 \%$ \\
\hline No & 0 & $0 \%$ \\
\hline Respuestas obtenidas & 21 & \\
\hline
\end{tabular}

Fuente: Elaboración propia.

Este resultado pudiera responder a las expectativas personales en donde Bandura (2001) destaca la autorrealización en la escogencia de la carrera universitaria en coherencia con el desempeño laboral.

Tabla 7. La carrera que está estudiando lo convierte en agente de cambio.

\begin{tabular}{|l|c|c|}
\hline Opciones & Frecuencia & Porcentaje \\
\hline Sí & 21 & $100 \%$ \\
\hline No & 0 & $0 \%$ \\
\hline Respuestas obtenidas & 21 & \\
\hline
\end{tabular}

Fuente: Elaboración propia.

Vivat Academia. Revista de Comunicación. 15 septiembre 2019 /15 diciembre 2019, nº 148, 57-76 
Castañón Octavio, N.; Ocanto Gómez, L., y Tirado Aldana, V. Estudio sobre expectativas laborales de estudiantes de la carrera de educación inicial en la Universidad Metropolitana de Caracas-Venezuela

Ante estos resultados se pudiera inferir que estudiar la carrera de Educación Inicial responde a expectativas e intereses personales, como pudiera ser una reacción social positiva de la profesión docente.

Tabla 8. Considera que necesita una titulación de Educación Inicial para desempeñarse como docente.

\begin{tabular}{|l|c|c|}
\hline Opciones & Frecuencia & Porcentaje \\
\hline Sí & 17 & $80,95 \%$ \\
\hline No & 4 & $19,05 \%$ \\
\hline Respuestas obtenidas & 21 & \\
\hline
\end{tabular}

Fuente: Elaboración propia.

Según los resultados reflejados en la tabla se evidencia que la mayoría de los sujetos consideran que la formación universitaria sí es necesaria para el desempeño docente. Cabe destacar que según Juárez y García (2015) el 47,74\% de los docentes a nivel nacional no poseen titularidad en el cargo que desempeñan.

Tabla 9. El desempeño como docente es asequible para su situación económica.

\begin{tabular}{|l|c|c|}
\hline Opciones & Frecuencia & Porcentaje \\
\hline Sí & 11 & $52,38 \%$ \\
\hline No & 10 & $47,62 \%$ \\
\hline Respuestas obtenidas & 21 & \\
\hline
\end{tabular}

Fuente: Elaboración propia.

Los datos obtenidos demuestran una disyuntiva entre los estudiantes. El empobrecimiento de los salarios y devaluación constante de los ingresos son determinantes para la profesión docente (Rodríguez, 2004), de allí la marcada diferencia de la respuesta de los sujetos.

Tabla 10. Condiciones laborales.

\begin{tabular}{|l|c|c|}
\hline Opciones & Frecuencia & Porcentaje \\
\hline Contrato a tiempo determinado & 19 & $90,48 \%$ \\
\hline Contrato temporal & 1 & $4,76 \%$ \\
\hline Acuerdo verbal & 1 & $4,76 \%$ \\
\hline Suplencia sin contrato & 0 & 0 \\
\hline Respuestas obtenidas & 21 & \\
\hline
\end{tabular}

Fuente: Elaboración propia.

Vivat Academia. Revista de Comunicación. 15 septiembre 2019 /15 diciembre 2019, n 148, 57-76 
Castañón Octavio, N.; Ocanto Gómez, L., y Tirado Aldana, V. Estudio sobre expectativas laborales de estudiantes de la carrera de educación inicial en la Universidad Metropolitana de Caracas-Venezuela

Los datos suministrados constatan las preferencias sobre las condiciones laborales que a pesar de ingresar al campo laboral con poca experiencia los estudiantes tienen proyecciones altas expresando expectativas materiales definidas.

Tabla 11. Beneficios.

\begin{tabular}{|l|c|c|}
\hline Opciones & Frecuencia & Porcentaje \\
\hline Cursos de actualización & 17 & $80,95 \%$ \\
\hline Incentivos de rendimiento y desempeño & 15 & $71,43 \%$ \\
\hline Bonos de alimentación generales: transporte, gimnasio, & 10 & $47,62 \%$ \\
\hline $\begin{array}{l}\text { Beneficios ger } \\
\text { guardería, entre otros. }\end{array}$ & 6 & $28,57 \%$ \\
\hline Descuento para eventos sociales & 3 & $14,29 \%$ \\
\hline Respuestas obtenidas & 51 & \\
\hline
\end{tabular}

Fuente: Elaboración propia.

A través de estos resultados se reitera las expectativas materiales acompañadas en menor medida de expectativas personales.

Tabla 12: Desempeño y país.

\begin{tabular}{|l|c|c|}
\hline Opciones & Frecuencia & Porcentaje \\
\hline Sí, en Venezuela & 7 & $33,33 \%$ \\
\hline Sí, fuera de Venezuela & 13 & $61,90 \%$ \\
\hline No & 1 & $4,76 \%$ \\
\hline Respuestas obtenidas & 21 & \\
\hline
\end{tabular}

Fuente. Elaboración propia.

El elevado número de sujetos que desean desempeñar su profesión en otro país pudiera responder a que en Venezuela, en la actualidad, no brinda poder adquisitivo, bienestar físico u oportunidades de superación laboral a sus habitantes, por tanto las expectativas no están satisfechas (Suárez, 2016).

De estos resultados se puede desprender las siguientes inferencias o relaciones:

- Al observar la "lista de entrada" en la que los estudiantes ingresaron a la universidad (si entran en "lista 1" se refiere a alto rendimiento hasta llegar a "lista 5" que se refiere al menor rendimiento), y se compara con las 5 primeras categorías en las que se dividió las respuestas del Índice Académico Acumulado (IAA), se puede observar que los estudiantes se mantuvieron con un índice correspondiente a su lista de ingreso, en la mitad de los casos incluso se encuentran actualmente en una categoría inferior del índice académico de la que corresponde a su lista.

Vivat Academia. Revista de Comunicación. 15 septiembre 2019 /15 diciembre 2019, n 148, 57-76 
Castañón Octavio, N.; Ocanto Gómez, L., y Tirado Aldana, V. Estudio sobre expectativas laborales de estudiantes de la carrera de educación inicial en la Universidad Metropolitana de

Caracas-Venezuela

La docencia en distintos niveles del sistema escolar forma parte de la respuesta del $95,24 \%$ (20) acerca de las oportunidades de trabajo que aspiran, lo cual llama la atención al observar que el $95,24 \%$ considera que en la sociedad venezolana no valora su carrera, y que el 100\% (21) piensan que estudiarla puede ayudarle a convertirse en un agente de cambio social, por lo que se puede indicar que a pesar de la poca valoración social de la carrera, los sujetos de la muestra siguen estudiando, desean desempeñarse en el ámbito educativo y aprovechar la carrera para impactar de manera positiva en la sociedad.

- Puesto a que, el 100\% (21) de los encuestados respondió que le gustaría continuar con su formación profesional, se destaca la coherencia que existe con el 80,95\% (17), que quisiera contar con cursos de actualización como un beneficio no remunerado, aún por encima de bonos de alimentación y beneficios generales como lo es el transporte.

- Los estudiantes demostraron coherencia entre los estudios que están realizando y sus deseos laborales, obteniendo que el 100\% (21) de la población considera que sus estudios en Educación Inicial le brindarán la oportunidad de conseguir su trabajo anhelado, demostrando la satisfacción de sus expectativas personales y materiales.

Se puede observar cómo los estudiantes ingresados en los años 2014 y 2016, que constituyen el 61,90\% (13), es decir, la mayor parte de la población, se visualizan como gerentes educativos y docentes en diferentes niveles. Cabe destacar que los que aspiran a cargos directivos y de mayor jerarquía, ingresaron años antes que los que aspiran ser docentes. En concordancia con Caram et al., (2009), las expectativas de quienes recién ingresaron a la carrera serán distintas de los que se encuentran cursando los últimos períodos académicos.

- El nivel de expectativas materiales, sociales y personales, en los cargos directivos representan mayor remuneración salarial, aceptación social y autorrealización, esto viene dado por su ubicación en la jerarquía de las organizaciones educativas.

- Al comparar los resultados de la perspectiva sobre sí para desempeñar esta profesión es necesario obtener una titulación y el país en el que desea ejercer la profesión, el $81 \%$ (17) respondió de manera afirmativa a la premisa de requerir una titulación, de ellos, doce (12) indicaron que tienen intención de tener su futuro laboral en un país distinto a Venezuela.

- A pesar de no poder observar una relación clara entre el estrato socioeconómico y la remuneración económica deseada, se observó en la remuneración salarial que del 61,9\% (13), que señaló su interés de ejercer la profesión en un país distinto a

Vivat Academia. Revista de Comunicación. 15 septiembre 2019 /15 diciembre 2019, n 148, 57-76 
Castañón Octavio, N.; Ocanto Gómez, L., y Tirado Aldana, V. Estudio sobre expectativas laborales de estudiantes de la carrera de educación inicial en la Universidad Metropolitana de

Caracas-Venezuela

Venezuela, ocho (8) encuestados indicaron desear la remuneración más alta, de 10 veces del salario mínimo y más de 10 veces el salario mínimo.

- No se observó relación directa entre el índice académico acumulado y las expectativas de futuros cargos de los encuestados.

- No se logró observar relación alguna entre la perspectiva de si el desempeño de la profesión es asequible para su situación económica y la remuneración a la que aspiran.

\section{DISCUSIÓN}

En la presente investigación, se puede concluir que existe diversidad de expectativas materiales, sociales y personales en los estudiantes de la carrera de Educación Inicial en la Universidad Metropolitana. Englobando todas éstas en sus expectativas laborales como recién egresados de la profesión, con escasa o ninguna experiencia laboral previa.

Atendiendo el primer objetivo específico: Detectar las expectativas laborales de los estudiantes de la carrera Educación Inicial de la Universidad Metropolitana, según las características personales. Se obtuvo que:

En los resultados obtenidos mediante la investigación, se conocieron los intereses personales de cada estudiante, demostrando variedad de criterios. Destacando, como respuesta generalizada, el deseo de convertirse en agente de cambio y desempeñar su rol docente $(90,24 \%)$ a pesar de considerar que la carrera no es valorada por la sociedad venezolana. Reafirmando que existe mayor influencia de sus expectativas personales a la hora de generar aspiraciones laborales, las expectativas sociales son minimizadas por los estudiantes.

Por otra parte, realizar un sondeo acerca de los niveles de ingreso que perciben los estudiantes, fue significativo para la investigación, ya que autores sustentan que mientras más conocimiento y aceptación del mismo, más ajustadas serán sus expectativas laborales. Asimismo, se identifica una población entre estratos B y C en su mayoría. No existen grandes diferencias entre las oportunidades de trabajo que desean los estudiantes y su estrato socioeconómico.

Sin embargo, se considera beneficioso realizar dichos estudios, debido a que, ciertamente los encuestados lograron ubicarse en el estrato correspondiente, tomando en cuenta sus condiciones de vivienda y ocupación del responsable económico. Al mismo tiempo, se evidenció que el 47,62\% puntualizó como oportunidad trabajo deseado la gerencia educativa. Para las autoras, significa que sus expectativas materiales, sociales y personales son altas, por cuanto se proyectan en el escalafón más

Vivat Academia. Revista de Comunicación. 15 septiembre 2019 /15 diciembre 2019, n 148, 57-76 
Castañón Octavio, N.; Ocanto Gómez, L., y Tirado Aldana, V. Estudio sobre expectativas laborales de estudiantes de la carrera de educación inicial en la Universidad Metropolitana de Caracas-Venezuela

alto de la jerarquía educativa, es decir, poseen expectativas ajustadas a su profesión, pero en una alta gama.

Sumado a esto, la perspectiva de los estudiantes con base a la necesidad de poseer un título en Educación para poder ejercer la profesión, el 80,95\% considera necesaria la titulación, pudiendo estimarse como un nivel alto en la valoración del empleo

En consonancia con las características personales, los estudiantes demostraron, al momento de escoger con libertad su área adicional de trabajo, una tendencia hacia ramas tradicionales que complementan la Educación Inicial, siendo éstas, psicología y psicopedagogía en un $42,86 \%$ de la población.

También, los estudiantes probaron sus deseos individuales de seguir formándose mediante talleres, masters, postgrados y doctorados, siendo una tendencia del $100 \%$ de la muestra, puesto que, las autoras infieren, que poseen deseos de expandir sus retribuciones económicas, prestigio y autorrealización. Dichos resultados, se consideraron relevantes al verificar las expectativas materiales de los mismos, ya que como lo afirma la literatura, el mercado laboral es dinámico, cambiante y altamente demandante, por lo cual, los profesionales deben contar con formación continua y actualizada. A la luz de tal afirmación, los encuestados, consideraron una prioridad dentro de los beneficios laborales, los cursos de actualización $(80,95 \%)$, dato relevante al considerar la situación de Venezuela, a nivel de alimentación y necesidades monetarias.

De ahí que, prosiguiendo con las expectativas materiales obtenidas, existió una diferencia donde los resultados arrojan que el 52,58\% de los estudiantes considera que con el título de Licenciado en Educación Inicial podrán tener una situación económica estable y en su mayoría en instituciones privadas, ya que las mismas responden a las necesidades socioeconómicas de los futuros egresados.

Por consiguiente, al realizar dicha escogencia, los estudiantes demuestran, nuevamente, expectativas materiales y sociales elevadas, al seleccionar diez veces el salario mínimo como remuneración adecuada para un educador recién egresado $(38,10 \%)$. La mayoría de los encuestados desearía poseer condiciones laborales con remuneraciones y beneficios como los señalados anteriormente.

En cuanto al segundo objetivo específico, se tiene: Caracterizar las expectativas laborales de los estudiantes de la carrera Educación Inicial de la Universidad Metropolitana, según el perfil académico.

La mayoría de los encuestados iniciaron sus estudios en el año 2016, conformando la población más joven en Educación Inicial en la Universidad, las autoras consideran que

Vivat Academia. Revista de Comunicación. 15 septiembre 2019 /15 diciembre 2019, n 148, 57-76 
Castañón Octavio, N.; Ocanto Gómez, L., y Tirado Aldana, V. Estudio sobre expectativas laborales de estudiantes de la carrera de educación inicial en la Universidad Metropolitana de

Caracas-Venezuela

las oportunidades de trabajo podrían variar por dicha vigencia en la carrera. Sin embargo, el estudio demostró que dicha población posee expectativas similares a los que ingresaron en años anteriores. Es decir, el tiempo de formación y permanencia en los estudios académicos para esta investigación no fueron determinantes, lo cual va en contraposición con lo expuesto por los autores estudiados.

Con el objeto de, conocer a profundidad la trayectoria de los estudiantes, que poseen expectativas claramente delimitadas, se confirmó la vía de ingreso de los mismos, donde la mayoría accedió mediante el examen de diagnóstico, ubicación y evaluación integral $(80,95 \%)$.

Como resultado, dichos estudiantes que se posicionaron en ciertas listas de ubicación (1 a la 5), continúan con índices académicos similares a las listas obtenidas, e inclusive disminuyeron su rendimiento. Siendo un resultado adicional de gran interés, al no observarse movilidad en cuanto a su progreso académico dentro de la formación básica y específica de la Institución.

Ahora bien, gran parte de la población considera como su oportunidad de trabajo fundamental a la docencia. Pero, un resultado que genera disparidad, es el obtenido en la valoración de las competencias específicas, ya que la competencia que recibió mayor puntaje fue la que responde al docente como agente de cambio social, en tres dimensiones: educativa, legal y cultural (63 puntos en total). Los estudiantes no otorgaron la mayor valoración a la competencia que por excelencia sustenta el rol docente, la planificación de la educación (46) y la evaluación (48). Al mismo tiempo, se evidencia congruencia con su perfil personal, acerca de la carrera de Educación Inicial como posible agente de cambio para la sociedad venezolana.

El tercer objetivo específico, responde a: Explorar las expectativas laborales de los estudiantes de la carrera Educación Inicial de la Universidad Metropolitana, según el contexto país.

Ciertamente, como lo afirman las autoras, la migración venezolana es una situación inminente, siendo reflejada en la población de estudiantes de Educación Inicial con un énfasis notable $(61,90 \%)$, en coherencia con sus deseos de ejercer la docencia, pero trasladándose a países como España, Estados Unidos y Argentina. De igual modo, fue notorio que parte de la población, aunque fue la minoría (33,33\%), desea desempeñarse como educador en Venezuela, considerándose llamativo su porcentaje.

Finalmente el determinar las expectativas laborales de los estudiantes de la carrera de Educación Inicial en la UNIMET, otorga una valoración fundamental para contribuir al proceso de mejoramiento continuo de la calidad académica, puesto que, desde el conocimiento de las realidades, intereses, necesidades y evidentemente, las tres

Vivat Academia. Revista de Comunicación. 15 septiembre 2019 /15 diciembre 2019, n 148, 57-76 
Castañón Octavio, N.; Ocanto Gómez, L., y Tirado Aldana, V. Estudio sobre expectativas laborales de estudiantes de la carrera de educación inicial en la Universidad Metropolitana de

Caracas-Venezuela

expectativas que se estudiaron en la presente investigación, se pueden elaborar planes de acción funcionales, en pro de la excelencia académica y laboral de la población unimetana, y el dinamismo del mercado laboral.

\section{REFERENCIAS}

Banco Mundial (2017b). Venezuela. Recuperado de: www.datos.bancomundial.org/pais/venezuela

Bandura, A. (2001). Guía para la construcción de escalas de autoeficacia. Recuperado de: www.uky.edu/ eushe2/Pajares/effguideSpanish.html

Balestrini, M. (2002). Cómo se elabora el proyecto de investigación. BL Consultores asociados: Caracas.

Batlle, S.; Vidondo, M.; Dueñas, M.; Núñez, M., y Rodríguez, M. (2009). Expectativas y aspiraciones laborales de jóvenes que cursan primer año de la escuela media. Recuperado de: http://hdl.handle.net/10915/17320

Caram, G.; Gil, M., y Naigeboren, M. (2009). Motivación y expectativas laborales en alumnos universitarios. I Congreso Internacional de Investigación y Práctica Profesional en Psicología XVI Jornadas de Investigación Quinto Encuentro de Investigadores en Psicología del MERCOSUR. Recuperado de: https://www.aacademica.org/000$\underline{\text { 020/377.pdf }}$

Corica, A. (2012). Las expectativas sobre el futuro educativo y laboral de jóvenes de la escuela secundaria: entre lo posible y lo deseable. Última década, 36, 71-95. Recuperado de: https://scielo.conicyt.cl/pdf/udecada/v20n36/art04.pdf

Facultad de Ciencias Jurídicas y Políticas [FCJP] (2013). Anteproyecto de Ley de Educación Universitaria. Universidad del Zulia. Recuperado de: http://www.luz.edu.ve/index.php?option=com_content\&view=category\&id=103\&I temid $=508$

Farné, S. (2011). Observatorios del mercado de trabajo: ¿Qué son y cómo funcionan? Economía Institucional, 13(24), 429-445. Recuperado de: https://dialnet.unirioja.es/descarga/articulo/3668789.pdf

Fidias, A. (2012). El Proyecto de Investigación: Introducción a la metodología científica. Caracas: Episteme.

Vivat Academia. Revista de Comunicación. 15 septiembre 2019 /15 diciembre 2019, n 148, 57-76 
Castañón Octavio, N.; Ocanto Gómez, L., y Tirado Aldana, V. Estudio sobre expectativas laborales de estudiantes de la carrera de educación inicial en la Universidad Metropolitana de

Caracas-Venezuela

Guevara, M. (2014). Mi primera búsqueda de empleo. El proceso a conocer. Observatorio Laboral Revista Venezolana, 7(14), 35-49. Recuperado de: http://servicio.bc.uc.edu.ve/faces/revista/lainet/lainetv7n14/art02.pdf

Juárez, J., García, E. (2015). Contexto educativo y formación docente en Venezuela. Escuela de Educación Universidad Católica Andrés Bello. Foro Cerpe-Serie Educalidad, 8.

Hernández, R., Fernández, C., y Baptista, P. (2010). Metodología de la investigación. México D.F.: McGraw-Hill.

Pérez, C. (2015). Inserción laboral de universitarios desde la perspectiva psicosocial. Universidad de Extremadura.

Rodríguez, N. (2004). Retos de la formación de docentes en Venezuela. Revista Pedagógica, 25(73).

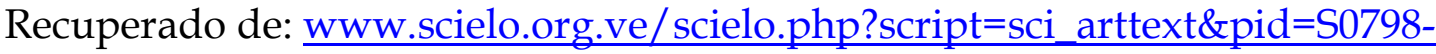
$\underline{97922004000200002}$

Sieglin, V., Zúñiga, M. (2010). Brain Drain en México: estudio sobre expectativas de trabajo y disposición hacia la migración laboral en estudiantes de Ingeniería y Ciencias Naturales. Perfiles educativos, 32(128), 55-79. Recuperado de: www.scielo.org.mx/pdf/peredu/v32n128/v32n128a4.pdf

Suárez, B. (2016). Éxodo intelectual en el ámbito venezolano: una mirada desde la percepción de los estudiantes universitarios. Venezuela en la mira de las ciencias económicas y sociales. Encuentro de saberes, 6, 1451-1462. Recuperado de: http://mriuc.bc.uc.edu.ve/bitstream/handle/123456789/4300/tomo6.pdf

Suriá, R., Rosser, A., y Villegas, E. (2013b). Diferencias en actitudes y expectativas de futuro laboral antes y después de haber iniciado estudios universitarios. XI Jornadas de redes de investigación en docencia universitaria. Recuperado de: web.ua.es/es/ice/jornadasredes/documentos/2013-comunicaciones-orales/334594.pdf

UNIMET. (s.f.). Educación. Recuperado de: www.unimet.edu.ve/educacion/

\section{AUTORAS}

\section{Natalia Castañón Octavio}

Profesora e investigadora a tiempo completo en la Universidad Metropolitana (1993-). Fundadora de tuterapiaenlinea.com (2014-). Licenciada en Educación (Universidad Metropolitana, 1991). Maestría en Psicología Escolar (Universidad Complutense de

Vivat Academia. Revista de Comunicación. 15 septiembre 2019 /15 diciembre 2019, nº 148, 57-76 
Castañón Octavio, N.; Ocanto Gómez, L., y Tirado Aldana, V. Estudio sobre expectativas laborales de estudiantes de la carrera de educación inicial en la Universidad Metropolitana de

Caracas-Venezuela

Madrid, 1993). Doctorada en Filosofía y Ciencias de la Educación (Universidad Complutense de Madrid, 1999). Ha realizado carrera docente, gerencial e investigación: jefa del departamento de Didáctica (2002-), jefa del departamento de Humanidades (2014-), gerente general del centro de aprendizaje en línea (2014) y profesora investigadora. Ha sido tutora de treinta y siete tesis de pregrado, maestría y doctorado. Publicaciones en veinte $\mathrm{y}$ dos revistas o libros arbitrados a nivel nacional e internacional. Ha participado en cuarenta congresos nacionales e internacionales con ponencias arbitradas.

ncastanon@unimet.edu.ve

Orcid ID: https:// orcid.org/0000-0002-7532-6924

\section{Laura Ocanto Gómez}

Es docente de inglés como lengua extranjera en el Colegio Integral El Ávila de Caracas (2018- ). Licenciada en Educación de la Universidad Metropolitana en el 2019 (CaracasVenezuela). Se desempeñó como coordinadora de RRSS de la Agrupación Generadores de Conocimiento, Universidad Simón Bolívar (2015-2016) y como asistente de investigación en el Departamento de Ciencias de la Educación (2018) junto con la profesora Natalia Castañón Octavio.

laura.ocanto@correo.unimet.edu.ve

\section{Valerie H. Tirado Aldana}

Actualmente trabaja como docente suplente en el Colegio Naval de Bogotá. Licenciada en Educación, mención Inicial de la Universidad Metropolitana (Caracas-Venezuela). Se desempeñó como coordinadora del centro de estudiantes de educación en la Universidad Metropolitana (2016-2019). Aspirante a estudiante de la Maestría de Psicopedagogía de la Universitat de Barcelona en su segunda cohorte (España). valerietirado@correo.unimet.edu.ve

Vivat Academia. Revista de Comunicación. 15 septiembre 2019 /15 diciembre 2019, nº 148, 57-76 